
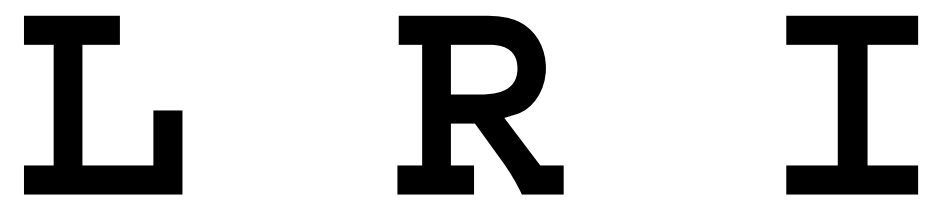

\title{
A NOTE ON k-WALKS IN BRIDGELESS
}

\section{GRAPHS}

KAISER T / KUZEL R / LI H / WANG G

Unité Mixte de Recherche 8623

CNRS-Université Paris Sud - LRI

$04 / 2006$

Rapport de Recherche $N^{\circ} 1444$

\section{CNRS - Université de Paris Sud}

Centre d'Orsay

LABORATOIRE DE RECHERCHE EN INFORMATIQUE

Bâtiment 490

91405 ORSAY Cedex (France) 


\title{
A note on $k$-walks in bridgeless graphs
}

\author{
Tomáš Kaiser ${ }^{1} \quad$ Roman Kužel ${ }^{1} \quad \mathrm{Hao} \mathrm{Li}^{2,3,5}$ \\ Guanghui Wang ${ }^{2,4,5}$
}

\begin{abstract}
We show that every bridgeless graph of maximum degree $\Delta$ has a spanning $\lceil(\Delta+1) / 2\rceil$-walk. The bound is optimal.
\end{abstract}

\section{Introduction}

Following Jackson and Wormald [6], we define a $k$-walk in a graph $G$ to be a closed spanning walk visiting each vertex at most $k$ times, where $k \geq 1$ is an integer. Being an interesting variation on the notion of a Hamilton cycle, this concept has received considerable attention (see, e.g., $[2,3,5]$ ).

Our aim in this note is to determine the least possible $k=k(\Delta)$ such that every graph of maximum degree $\Delta$ admits a $k$-walk. For general graphs, this problem is trivial since a tree of maximum degree $\Delta$ has a $\Delta$-walk [6], and it clearly does not admit any $k$-walk with $k<\Delta$. The situation changes if we restrict ourselves to bridgeless (i.e., 2-edge-connected) graphs. We prove the following result:

Theorem 1. Every bridgeless graph of maximum degree $\Delta$ admits $a\lceil(\Delta+1) / 2\rceil$ walk.

Theorem 1 follows directly from a more general statement (Theorem 5) which we prove in Section 2. In Section 3, we complement this result by showing that the bound in Theorem 1 is best possible.

\footnotetext{
${ }^{1}$ Department of Mathematics and Institute for Theoretical Computer Science, University of West Bohemia, Univerzitní 8, 30614 Plzeň, Czech Republic. E-mail: \{kaisert,rkuzel\}@kma.zcu.cz. Supported by project 1M0545 and Research Plan MSM 4977751301 of the Czech Ministry of Education.

${ }^{2}$ Laboratoire de Recherche en Informatique, UMR 8623, C.N.R.S.-Université de Paris-Sud, 91405 Orsay cedex, France. E-mail: $\{1 i, w g h\} @ l r i . f r$.

${ }^{3}$ School of Mathematics and Statistics, Lanzhou University, 730000 Lanzhou, Gansu, China.

${ }^{4}$ School of Mathematics and System Science, Shandong University, 250100 Jinan, Shandong, China.

${ }^{5}$ Supported by the NSFC (60373012 and 10471078), SRSDP (20040422004) and PDSF (2004036402) of China.
} 


\section{The upper bound}

All the graphs we consider are finite and loopless, multiple edges are allowed. Throughout this section, $G$ is a graph. Its vertex set and the edge set are denoted by $V(G)$ and $E(G)$, respectively. If $W$ is a walk in $G$, we let $p_{W}(x)$ denote the number of times a vertex $x \in V(G)$ is visited by $W$. An edge-cut in $G$ is an inclusionwise minimal set of edges whose removal disconnects $G$.

Let $v$ be a vertex of $G$ and $e_{1}, e_{2}$ be two distinct edges incident with $v$. Let $v_{i}$ be the endvertex of $e_{i}$ distinct from $v$. We recall the operation of splitting $e_{1}$ and $e_{2}$ off $v$. The resulting graph $G\left(v, e_{1}, e_{2}\right)$ is defined to be $G$ with an added vertex $v^{*}$ and the edges $e_{1}, e_{2}$ replaced with $e_{1}^{*}, e_{2}^{*}$, where $e_{i}^{*}$ has ends $v^{*}$ and $v_{i}$. The following assertion is an easy consequence of Fleischner's Splitting Lemma [4] (see also [9, Theorem A.5.2]):

Lemma 2. Let $v$ be a vertex of degree at least 4 in a bridgeless graph $G$. There exist edges $e_{1}, e_{2}$ incident with $v$ such that the graph $G\left(v, e_{1}, e_{2}\right)$ is bridgeless.

Lemma 3. Let $v$ be a vertex of a graph $G$, let $e_{1}, e_{2}$ be two edges incident with $v$, and $H=G\left(v, e_{1}, e_{2}\right)$. If $W$ is a spanning closed walk in $H$ such that $p_{W}\left(v^{*}\right) \leq 2$ (where $v^{*}$ is defined as above), then $G$ admits a closed walk $\tilde{W}$ such that

(i) for all $z \in V(G) \backslash\{v\}, p_{\tilde{W}}(z) \leq p_{W}(z)$, and

(ii) $1 \leq p_{\tilde{W}}(v) \leq p_{W}(v)+1$.

Proof. Enumerate the vertices visited by $W$ as

$$
W=x_{0} x_{1} \ldots x_{\ell}
$$

where $x_{0}=x_{\ell}$. Any operations on the indices of the vertices in $W$ are performed modulo $\ell$. A subwalk of $W$ is a walk of the form

$$
\left[x_{i}, x_{j}\right]=x_{i} x_{i+1} \ldots x_{j-1} x_{j} .
$$

We write $\left[x_{i}, x_{j}\right]^{-}$for the reverse subwalk $x_{i} x_{i-1} \ldots x_{j+1} x_{j}$.

If $p_{W}\left(v^{*}\right)=1$, then we may set $\tilde{W}=W$. Thus, it may be assumed that $p_{W}\left(v^{*}\right)=2$. Let the two occurences of $v^{*}$ in $W$ be $x_{i}$ and $x_{j}$, where $i<j$. We use the symbols $v_{1}, v_{2}$ as introduced in the definition of splitting.

Suppose first that both neighbors of $x_{i}$ on $W$ coincide with $v_{1}$, i.e., $\left[x_{i-1}, x_{i+1}\right]=$ $v_{1} v^{*} v_{1}$. Then we may set

$$
\tilde{W}=\left[x_{0}, x_{i-1}\right]\left[x_{i+2}, x_{j-1}\right] v\left[x_{j+1}, x_{\ell}\right]
$$

(see Figure 1a). Note that we may indeed concatenate the subwalks $\left[x_{0}, x_{i-1}\right]$ and $\left[x_{i+2}, x_{j-1}\right]$ since $x_{i+2}$ is a neighbor of $x_{i-1}=x_{i+1}$. It is easy to check that $\tilde{W}$ satisfies the conditions (i)-(ii). By symmetry, we may assume that the neighbors 
of $x_{i}$ on $W$ are $v_{1}$ and $v_{2}$, and the same holds for $x_{j}$. We now distinguish two cases.

Case 1: $\left[x_{i-1}, x_{i+1}\right]=\left[x_{j-1}, x_{j+1}\right]=v_{1} v^{*} v_{2}$. We set

$$
\tilde{W}=\left[x_{0}, x_{i-1}\right]\left[x_{j-2}, x_{i+2}\right]^{-}\left[x_{j+1}, x_{\ell}\right]
$$

(see Figure 1b). Note that the conditions (i)-(ii) are satisfied. By symmetry, this case also covers the possibility that $\left[x_{i-1}, x_{i+1}\right]$ and $\left[x_{j-1}, x_{j+1}\right]$ equal $v_{2} v^{*} v_{1}$.

Case 2: $\left[x_{i-1}, x_{i+1}\right]=\left[x_{j-1}, x_{j+1}\right]^{-}=v_{1} v^{*} v_{2}$. Since $W$ is spanning, there is $k$ such that $x_{k}=v$. We may assume that $i<k<j$ since the other possibility $(k<i$ or $k>j)$ is symmetric. The walk

$$
\tilde{W}=\left[x_{0}, x_{i-1}\right] v\left[x_{k-1}, x_{i+2}\right]^{-}\left[x_{j-1}, x_{k+1}\right]^{-} v\left[x_{j+1}, x_{\ell}\right]
$$

(see Figure 1c for an illustration) meets the requirements.

Since we have covered, up to symmetry, all the possibilities, the proof is complete.

Spanning closed walks correspond to edge weight functions in the following straightforward way. Let $w$ be a function assigning to each edge $e \in E(G)$ a non-negative integer $w(e)$. For any set $X \subset E(G)$, we define

$$
w(X)=\sum_{e \in X} w(e)
$$

The function $w$ is an Eulerian weight if for each edge-cut $C$ in $G$, the value $w(C)$ is positive and even. Note that if $w$ is an Eulerian weight, then each vertex $v$ must be incident with an edge of nonzero weight, since the set

$$
\partial v=\{e: e \text { is incident with } v\}
$$

contains an edge-cut.

Lemma 4. Let $G$ be a graph and $k \geq 1$ a positive integer. The graph $G$ has a $k$-walk if and only if it admits an Eulerian weight $w$ such that for each $v \in V(G)$,

$$
w(\partial v) \leq 2 k
$$

Proof. If $G$ has a $k$-walk $W$, then the function assigning each edge the number of times it is traversed by $W$ (in any direction) is clearly an Eulerian weight satisfying (1). Conversely, let $w$ be such an Eulerian weight. Replacing each edge $e$ by $w(e)$ parallel edges (or deleting it if $w(e)=0$ ), we obtain a (connected) Eulerian graph of maximum degree at most $2 k$. Any Euler trail in the new graph determines a $k$-walk in $G$.

We now proceed to prove the main result of this paper. 

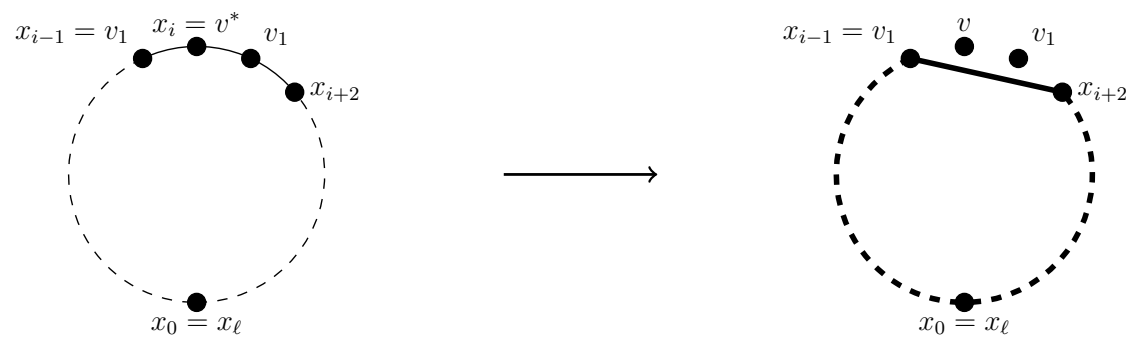

(a) $W$ contains a subwalk $v_{1} v^{*} v_{1}$.
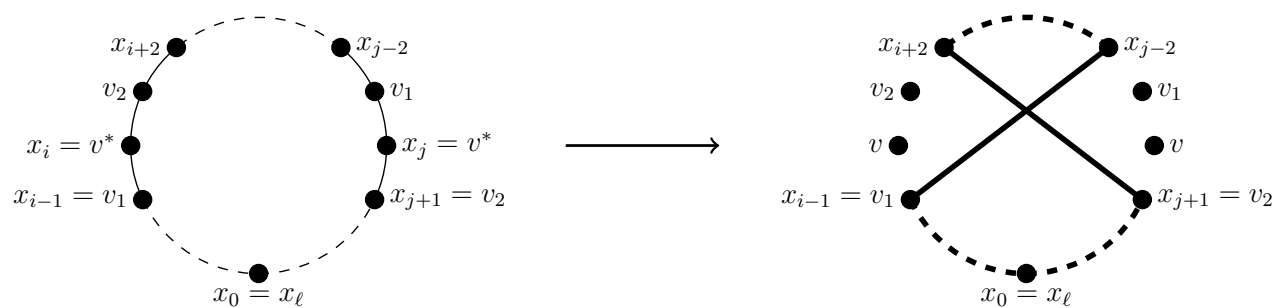

(b) $W$ contains two subwalks $v_{1} v^{*} v_{2}$.
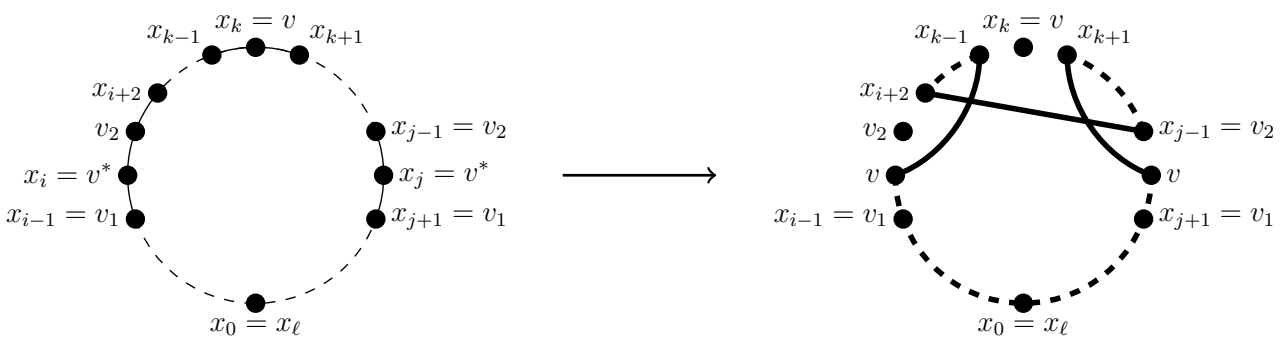

(c) $W$ contains subwalks $v_{1} v^{*} v_{2}$ and $v_{2} v^{*} v_{1}$.

Figure 1: The possibilities considered in the proof of Lemma 3. Dashed lines represent walks, edges are drawn solid. In each case, thick lines give the resulting walk $\tilde{W}$ in $G$. 
Theorem 5. Every bridgeless graph admits a closed spanning walk $W$ such that for each vertex $x$,

$$
p_{W}(x) \leq\left\lceil\frac{\operatorname{deg}(x)+1}{2}\right\rceil .
$$

Proof. By induction. We first establish the assertion for graphs with maximum degree $\Delta \leq 3$. Then, we prove that if $\Delta(G) \geq 4$, the assertion holds for $G$ whenever it holds for all bridgeless graphs that are smaller than $G$ in a certain sense.

Assume first that $\Delta(G) \leq 3$. Since the minimum degree is at least 2 and the claim is clearly true if $G$ is a circuit, we may assume that $G$ is a subdivision of a cubic bridgeless graph $H$. By the well-known Petersen theorem (see, e.g., [1, Corollary 2.2.2]), $H$ has a 1-factor $F$. Let $w: E(G) \rightarrow\{1,2\}$ be a function whose value $w(e)$ is 2 if the edge of $H$ corresponding to $e$ belongs to $F$, and 1 otherwise. It is easy to see that $w$ is an Eulerian weight in $G$. By Lemma $4, G$ admits a 2-walk.

Next, assume that $\Delta(G) \geq 4$ and (2) holds for all graphs $G^{\prime}$ such that either $\Delta\left(G^{\prime}\right)<\Delta(G)$, or $\Delta\left(G^{\prime}\right)=\Delta(G)$ and $G^{\prime}$ has fewer vertices of maximum degree. We show that the assertion holds for $G$.

Let $v$ be any vertex of degree $\Delta(G)$. Lemma 2 ensures that there are two edges $e_{1}, e_{2}$ such that $G\left(v, e_{1}, e_{2}\right)$ is bridgeless. Since the resulting graph has fewer vertices of degree $\Delta(G)$, the induction hypothesis implies that $G\left(v, e_{1}, e_{2}\right)$ admits a closed spanning walk $W_{0}$ satisfying (2). Using Lemma 3, we find a closed spanning walk $\tilde{W}_{0}$ in $G$ such that for each vertex $x \in V(G) \backslash\{v\}, p_{\tilde{W}_{0}}(x) \leq p_{W_{0}}(x)$, and

$$
1 \leq p_{\tilde{W}_{0}}(v) \leq p_{W_{0}}(v)+1
$$

Clearly, the closed walk $\tilde{W}_{0}$ in $G$ is spanning, satisfies (2) at all vertices $x \neq v$, and

$$
p_{\tilde{W}_{0}}(v) \leq p_{W_{0}}(v)+1 \leq\left\lceil\frac{(\operatorname{deg}(v)-2)+1}{2}\right\rceil+1=\left\lceil\frac{\operatorname{deg}(v)+1}{2}\right\rceil .
$$

It follows that $W=\tilde{W}_{0}$ satisfies $(2)$ at all vertices of $G$.

\section{The lower bound}

Theorem 6. For every even $\Delta \geq 4$, there is a 2-connected graph $G$ with $\Delta(G)=$ $\Delta$ and no $(\Delta / 2)$-walk.

Proof. Let $k=\Delta-1$. For $i \in\{1, \ldots, 9\}$, take a copy $H_{i}$ of the complete bipartite graph $K_{2, k}$, with the degree $k$ vertices denoted by $a_{i}$ and $b_{i}$.

The graph $G$ is obtained from the disjoint union of the graphs $H_{1}, \ldots, H_{9}$ by adding new vertices $a$ and $b$, together with edges

$$
\left\{a a_{i}: i \in\{1,4,7\}\right\} \cup\left\{b b_{i}: i \in\{3,6,9\}\right\} \cup\left\{b_{i} a_{i+1}: i \in\{1,2,4,5,7,8\}\right\}
$$




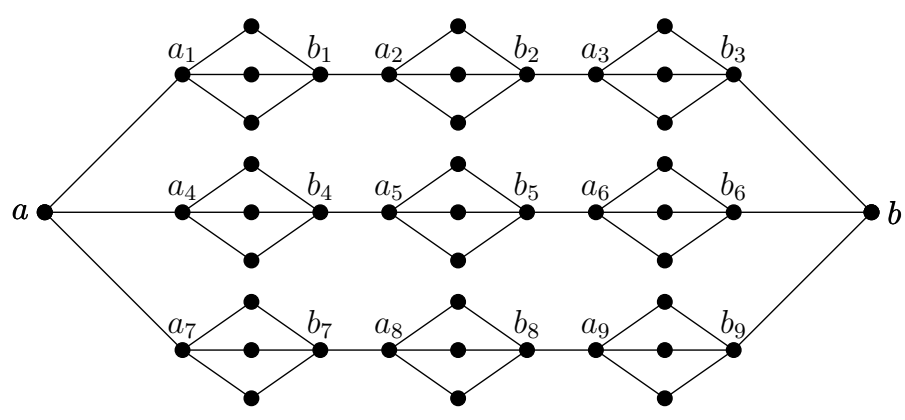

Figure 2: A 2-connected graph with maximum degree 4 and no 2-walk.

(see Figure 2 for an illustration with $k=3$ ). Note that the maximum degree of $G$ is $k+1=\Delta$.

We now show that $G$ has no $(\Delta / 2)$-walk. Assume the contrary. By Lemma 4 , there is an Eulerian weight $w$ satisfying

$$
w(\partial v) \leq \Delta
$$

for each vertex $v$.

Since $w(\partial a)$ is even, there is an edge incident with $a$ that receives an even value. We may assume that $w\left(a a_{1}\right)$ is even. Since each pair of edges from the set

$$
C=\left\{a a_{1}, b_{1} a_{2}, b_{2} a_{3}, b_{3} b\right\}
$$

forms an edge-cut, at most one edge $e \in C$ has $w(e)=0$. Consequently, for some $i \in\{1,2,3\}$, both edges in $C$ that are incident with either $a_{i}$ or $b_{i}$ are assigned a positive even value by $w$. Let $C_{i}$ be the set consisting of these two edges. We have

$$
w\left(E\left(H_{i}\right)\right)=w\left(\partial a_{i}\right)+w\left(\partial b_{i}\right)-w\left(C_{i}\right) \leq 2 \Delta-4
$$

by (3).

For each vertex $d$ of degree 2 in $H_{i}, \partial d$ is an edge-cut, whence $w(\partial d) \geq 2$. It follows that

$$
w\left(E\left(H_{i}\right)\right) \geq 2 k=2 \Delta-2,
$$

contradicting (4). It follows that $G$ does not admit any $(\Delta / 2)$-walk.

Recall that a trail in a graph is a walk using each edge at most once. By a well-known result of Jaeger [7,8], every 4-edge-connected graph $G$ admits a spanning closed trail. It is easy to see that if the maximum degree of $G$ is $\Delta$, then such a trail gives rise to a $\lceil\Delta / 2\rceil$-walk in $G$. For even $\Delta$, this improves on the bound of Theorem 1 by one. Since the tightness example constructed in the proof of Theorem 6 makes a heavy use of edge-cuts of size 2, one may wonder whether such an improvement is possible even for 3-edge-connected graphs $G$. We leave this as an open problem: 
Problem 7. Does every 3-edge-connected graph of maximum degree $\Delta$ admit a $\lceil\Delta / 2\rceil$-walk?

\section{References}

[1] R. Diestel, Graph Theory, Springer-Verlag, New York, 2000.

[2] M. N. Ellingham, Spanning paths, cycles, trees and walks for graphs on surfaces, Surveys in graph theory (San Francisco, CA, 1995), Congr. Numer. 115 (1996), 55-90.

[3] M. N. Ellingham and X. Zha, Toughness, trees, and walks, J. Graph Theory 33 (2000), 125-137.

[4] H. Fleischner, Eine gemeinsame Basis für die Theorie der eulerschen Graphen und den Satz von Petersen, Monatsh. Math. 81 (1976), 267-278.

[5] Z. Gao, B. R. Richter and X. Yu, 2-walks in 3-connected planar graphs, Australas. J. Combin. 11 (1995), 117-122.

[6] B. Jackson and N. C. Wormald, $k$-walks in graphs, Australas. J. Combin. 2 (1990), 135-146.

[7] F. Jaeger, On nowhere-zero flows in multigraphs, Proceedings of the Fifth British Combinatorial Conference 1975, Congr. Numer. 15 (1975), 373-378.

[8] F. Jaeger, Flows and generalized coloring theorems in graphs, J. Combin. Theory Ser. B 26 (1979), 205-216.

[9] C.-Q. Zhang, Integer Flows and Cycle Covers of Graphs, Dekker, New York, 1997. 